\title{
A review of hyperspectral remote sensing of crops
}

\author{
Liheng Xia ${ }^{1,2,3,4,5, *}$, Xueying $\mathrm{Wu}^{1,2,3,4,5}$ \\ ${ }^{1}$ Shaanxi Provincial Land Engineering Construction Group Co., Ltd. China \\ ${ }^{2}$ Institute of Land Engineering and Technology, Shaanxi Provincial Land Engineering Construction Group Co., Ltd. China \\ ${ }^{3}$ Key Laboratory of Degraded and Unused Land Consolidation Engineering, Ministry of Natural Resources China \\ ${ }^{4}$ Shaanxi Provincial Land Consolidation Engineering Technology Research Center China \\ ${ }^{5}$ Land Engineering Technology Innovation Center, Ministry of Natural Resources China
}

\begin{abstract}
With the development of space science and technology, various resource monitoring environmental satellites provide multi-platform, multi-spectral, multi-temporal and wide-range real-time information for the study of surface dynamic changes, and remote sensing technology has become a powerful technical means for human to study the earth's resources and environment, while high-resolution and hyperspectral remote sensing has become the main source for fruit tree growth monitoring and fruit quality detection acquisition. This paper has the following aspects to introduce the current situation of application of high-resolution and hyperspectral remote sensing data.
\end{abstract}

Key words: Hyperspectral Remote Sensing, Agricultural crops, Monitoring

\section{Introduction}

Remote sensing image classification is one of the most widely used methods for extracting spatial and temporal information of geological features. Remote sensing image classification plays a very important role in thematic mapping, classification of feature types and change monitoring. Remote sensing image classification is the process of classifying each image element into different feature types according to its spectral characteristics and spatial structure characteristics. It has developed from traditional unsupervised classification and parametric supervised classification to non-parametric supervised classification such as neural network classification, genetic algorithm, machine learning classification algorithm and the direction of multiple classifiers combined with integrated algorithms.

Hyperspectral remote sensing refers to the remote sensing technology that uses very narrow $(\lambda / 100)$ and continuous spectral channels for continuous measurement of features. Hyperspectral remote sensing technology is a new remote sensing technology that has been developed rapidly in recent decades, which is a comprehensive technology integrating detector technology, weak signal detection, precision optical machinery, information processing technology, and computer technology. Researchers have measured and analyzed the spectra of dried and mashed leaves of various plants in detail, and obtained about 42 absorption features corresponding to certain biochemical components in the spectral range of $0.4-2.4 \mu \mathrm{m}$, which provides the possibility of estimating the biochemical components of leaves and even plants by remote sensing means. Therefore, hyperspectral remote sensing technology is widely used in agricultural crop growth monitoring[1]-[4].

In recent years, Unmannedaerialvehicle (UAV) technology has emerged as a bridge between surface measurement and satellite remote sensing. With its small size, light mass, low operating cost, high flexibility, realtime image transmission, and the ability to detect in special areas, it has played an important role in ecological environment monitoring, urban construction and planning, agricultural production, natural disaster monitoring during data It plays an irreplaceable role in real-time acquisition of data during ecological environment monitoring, urban construction and planning, agricultural production, natural disaster monitoring, etc. It is one of the important tools for high-resolution remote sensing data acquisition, and a useful supplement to ground observation (such as cameras) and high-altitude observation (such as remote sensing satellites). The application of UAV images in the field of agricultural remote sensing monitoring has great advantages and broad prospects. It has higher ground spatial resolution compared with satellite images and can bring additional remote sensing information such as fine texture of crops that satellite remote sensing does not have, and can be well applied in the field of fine agricultural remote sensing monitoring[5]-[8].

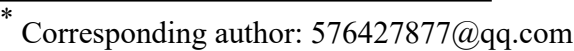




\section{Research on hyperspectral remote sensing in agriculture}

In agricultural production, hyperspectral remote sensing has been widely used in crop growth monitoring, yield estimation, quality monitoring, and pest and disease monitoring and prediction. At present, domestic and foreign research in remote sensing monitoring of crop diseases and pests mainly focuses on the spectral characteristics of crops subject to pest and disease stress and carries out feature extraction of pest and disease stress. Commonly used feature extraction methods include basic spectral operation and transformation methods, such as sensitive band extraction method, spectral vegetation index method, differential spectral analysis method, absorption valley (reflection peak and other spectral location parameters extraction method, etc.) and spectral data knowledge mining methods, such as principal component analysis, wavelet analysis, neural network method, support vector machine, partial least squares regression, etc. In order to carry out the research on the differentiation of different pests and diseases of crops, researchers have proposed novel spectral indices for disease identification. For example, by introducing RELIEF-F feature extraction algorithm, the spectral characteristics of wheat stripe rust, powdery mildew and aphids were elucidated, and new spectral indices (NSIs) for quantitative differentiation of specific diseases were constructed, including HI (Health-Index), PMI (PowderyMildew-Index), and YRI (YellowRust-Index). YRI (YellowRust-Index), and AI (Aphids-Index) [9], and these new vegetation indices were used for leaf and canopy-scale pest and disease differentiation with satisfactory results.

\subsection{Research on rice based on hyperspectral remote sensing}

\subsubsection{Research method}

In 2018, Yan Lin [10] obtained hyperspectral data spectral range of rice covering visible-near-infrared-short-wave infrared band, plants have unique absorption and reflection spectral characteristics in this range, and have different sensitivity to different vegetation on each band, so the correlation analysis between spectral data and crop physiological and biochemical parameters can be performed to find out the parameter in the effective range of spectral of the characteristic bands.

The correlation analysis of the spectral data with rice SPAD and leaf area index was carried out under MATLAB2014a environment using a self-programmed program, and the characteristic bands were selected according to the magnitude of the correlation coefficients. Statistical regression is the study of the relationship between the variation of a set of random variables $Y$ relative to another set of random variables $\mathrm{X}$. In hyperspectral remote sensing modeling, the physiological and biochemical parameters (e.g., leaf area index, chlorophyll content, leaf nitrogen content, water content, etc.) are usually taken as the dependent variables, and the hyperspectral data or their variation forms (e.g., original spectral reflectance and its derivative transformation, logarithmic transformation or spectral index) are taken as the independent variables, and the mathematical model based on the hyperspectral data is constructed by analyzing their variation patterns and mechanisms, and the physiological and biochemical parameters of vegetation are estimated theoretically. Theoretical estimation of vegetation physiological and biochemical parameters is carried out. In the modeling process, the total sample is usually divided into modeling samples and testing samples, which are used to support the construction of the model and the testing of the model accuracy, respectively.

\subsubsection{Hyperspectral characteristics of rice}

The structural and compositional information of different substances on the ground under the action of electromagnetic waves will form different absorption and reflection features in some specific bands within the electromagnetic spectrum, and such absorption and reflection features are usually referred to as the spectral features of the substances (Wang J. H. et al. 2008:1; Tong Q. H. et al. 2016:1) [11]-[12]. Such differences are precisely the important basis for remote sensing detection of the nature and shape of the features. In agricultural remote sensing, different vegetation or the same vegetation has different spectral characteristics at different growth and fertility stages, different production management measures, and different nutritional status, and the differences in the spectral characteristics of vegetation can accurately identify vegetation categories, monitor vegetation growth and development, estimate vegetation yield, and vegetation field management, which play an important role in the promotion of precision agriculture. In this chapter, the spectral characteristics of rice leaves and canopy will be discussed according to the factors affecting the spectral characteristics of rice, such as different nutritional status, different fertility stages, and different physiological and biochemical parameters.

\subsubsection{Hyperspectral estimation of rice SPAD at different fertility stages}

In 2018, Yan Lin et al. took rice at different fertility stages and firstly analyzed the changes of chlorophyll content of rice at different fertility stages, then calculated the correlation coefficients between the spectral reflectance and chlorophyll content of rice canopy at different fertility stages to establish a model for estimating chlorophyll content of rice based on spectral index and BP neural network, and used the coefficient of determination (R2), relative error (RE) and root mean square error ( RMSE) to validate the model and lay the foundation for hyperspectral monitoring of chlorophyll content in rice. 


\subsubsection{Estimation of leaf area index of rice based on hyperspectral remote sensing}

LeafAreaIndex (LAI), which refers to the total leaf area of vegetation per unit area, is an important functional evaluation parameter of vegetation canopy and plays a very important role in vegetation research, providing structured and quantitative information on the energy exchange between vegetation canopy and the environment (Huifengming et al. 2002; Fang Xiuqin et al. 2003; Li Kaili et al. 2005. Xie Qiaoyun et al. 2014) [13][16]. Leaf area index achieves control of physiological and biochemical processes such as photosynthesis, respiration, transpiration and charcoal cycling of vegetation by affecting vegetation light uptake, and can estimate vegetation net primary productivity more accurately, as well as effectively characterize vegetation growth and development and nutrient status and roughly estimate vegetation yield. The determination of leaf area index by traditional methods (area coefficient method, direct weighing method and square grid calculation method, etc.) has the shortcomings of large workload, time consuming and inability to perform large area measurement. The development of hyperspectral remote sensing technology has provided a reliable way for the non-destructive and rapid large-scale determination of vegetation leaf area index (LAI), and therefore the estimation of LAI using hyperspectral remote sensing has become an important area of research for scholars. When vegetation is photosynthesizing, chlorophyll strongly absorbs visible light and strongly reflects and scatters near-infrared light, so the reflectance of vegetation in visible and near-infrared bands contains a lot of information of canopy leaves. This unique spectral characteristic of vegetation is the basis for remote sensing quantitative analysis of leaf area index.

In 2018, Yan Lin et al. analyzed the variation of leaf area index of rice at different fertility periods as well as different fertilization levels to analyze the correlation between the spectral reflectance of rice canopy and leaf area index at each fertility period by selecting the spectral bands with the strongest correlation with leaf area index within each fertility period of rice, and constructed a model for estimating leaf area index at each fertility period of rice based on the characteristic bands by calculating the By calculating the correlation coefficients of six types of spectral indices and leaf area index in the range of 350-1000 nm, the BP neural network-based leaf area index estimation model for each fertility period was established by selecting the band combination with the highest correlation coefficient.

\subsection{Research based on hyperspectral remote sensing of maize}

\subsubsection{Research Methods}

In 2018, Wang Shuo [17] used two methods, traditional regression method and BP artificial neural network (BackPropagationNeuralNetwork, BPNN), to model the inversion of leaf chlorophyll content and leaf nitrogen content in maize canopy. The traditional regression methods mainly construct linear regression models and nonlinear regression models, and the nonlinear models include exponential models, polynomial models, logarithmic models, and power function models.

Artificial neural network is similar to the human brain nervous system, which is composed of many artificial neurons in parallel, so the amount of information that can be stored is huge, which makes artificial neural network can deal with problems with uncertainties and become a simple and effective means to deal with complex nonlinear problems. Artificial neural networks have high parallelism, fast processing speed and high fault tolerance; strong nonlinear mapping ability, capable of processing information by machine simulating human brain intelligence; strong self-learning and self-adaptive ability, as well as high fault tolerance, capable of solving problems that are difficult to be handled by mathematical models quickly and efficiently. In recent years, artificial neural networks are increasingly used in hyperspectral remote sensing research such as crop identification, monitoring crop growth, and crop biochemical parameter inversion with its above advantages (Luo Dan et al. 2016) [18].

To test the accuracy and reliability of the model, the coefficient of determination, root mean square error and relative error were selected as indicators for the modeling results, and the model estimates were fitted to the measured values by regression.

\subsubsection{Model for monitoring maize SPAD values based on hyperspectral remote sensing}

In 2018, Wang Shuo mainly studied canopy spectral reflectance and canopy leaf SPAD values, analyzed the changes of maize canopy leaf SPAD values at different fertility stages and the response characteristics of canopy spectral reflectance under different SPAD values, based on which, the spectra were first-order differential transformed, vegetation index and optimization index were constructed, and through correlation analysis, the SPAD values with greater correlation were selected Through the correlation analysis, we selected the original spectra, first-order differential spectra, vegetation indices and optimization indices, and constructed the SPAD estimation models based on the characteristic bands, vegetation indices, optimization indices and $\mathrm{BP}$ neural networks respectively, and verified the accuracy of each model, aiming to provide more accurate hyperspectral monitoring of the chlorophyll content of maize canopy in northwest China, and provide scientific basis for precision agriculture research and crop growth judgment.

\subsubsection{Hyperspectral estimation model of maize leaf nitrogen content}

Nitrogen transport is the most important management measure in crop production, and the right amount of nitrogen supply at the right time is the fundamental guarantee of high crop yield and quality, therefore, the production needs timely and reasonable nitrogen 
diagnosis and fertilization regulation based on timely grasp of crop growth and nitrogen status (Zhang et al. 2012) [19]. Leaf nitrogen content (LNC, \%) is one of the main indicators to characterize the nitrogen status of crop leaves, so the analysis of leaf nitrogen content changes is important for crop growth monitoring. The usual method for monitoring plant leaf nitrogen is laboratory Kjeldahl nitrogen determination, which is a complex, timeconsuming and inefficient operation process, and now, remote sensing monitoring using hyperspectral data has become the main choice for rapid, efficient and nondestructive monitoring of leaf nitrogen.

In 2018, Wang Shuo calculated the correlation between canopy spectra and maize leaf nitrogen content, and analyzed and compared the accuracy of models constructed based on characteristic bands, vegetation indices, optimized spectral indices, and BP neural networks, respectively, so as to provide a theoretical basis for nondestructive estimation of maize leaf nitrogen content and large-scale growth condition monitoring of maize.

\subsection{Hyperspectral remote sensing based wheat research}

\subsubsection{Research methods}

In 2017, $\mathrm{Li} \mathrm{Na}$ [20] selected 72 sets of these sample spectral data for modeling. (1) Correlation analysis of the agronomic parameters with the original spectral reflectance and first-order derivative spectral data under different sowing and nitrogen levels was conducted to screen the sensitive spectral band range of the agronomic parameters; (2) Based on the previous research results, correlation studies were conducted on the existing spectral parameter numbers and agronomic indicators, with the spectral index, which is closely related to the agronomic indicators, as the independent variable and the agronomic parameters as the dependent variables, to establish simple linear (3) to correlate the canopy chla $+b$ content with GPC, LAI and GY in each fertility period, so as to clarify the best fertility period for estimation, and interlink with the reflectance spectral data of the best fertility period to establish the GPC and GY estimation model of wheat based on spectral data. Independent samples of spectral data were selected to test the constructed models. Six indicators, correlation coefficient $(\mathrm{r})$, precision (R2), root mean square error (RMSE), relative error (RE), mean absolute error (da) and ratio of the mean of the observed values (dap), were used for comprehensive evaluation, and a 1:1 relationship between the observed and predicted values was plotted. The smaller the RMSE, RE, da and dap, and the larger the $r$ and R2, the greater the difference between the modelfitted values and the actual The smaller the difference between the measured values, the better the fitted model.

\subsubsection{Wheat canopy reflectance spectral characteristics analysis}

Wheat spectral reflectance characterizes the differences in the internal tissue structure and canopy layout of wheat. And the advancement of sowing amount, nitrogen application level and fertility period can significantly affect not only the canopy structure of wheat, but also the changes of the internal tissue structure of wheat (such as chlorophyll, etc.), which have important effects on the spectral characteristics. The experiments investigated the response characteristics of canopy spectral reflectance of $\mathrm{Pu}$ Bing 151 to seeding rate, nitrogen application and fertility period ( $\mathrm{Li} \mathrm{Na} 2017$ ).

\subsubsection{Wheat canopy chlorophyll content monitoring model}

Chla $+\mathrm{b}$ content is one of the good parameters to respond to photosynthetic capacity, growth and development of vegetation as well as $\mathrm{N}$ nutrition status, and real-time rapid and non-destructive estimation of chla $+\mathrm{b}$ content is of great significance for wheat nutrition evaluation and $\mathrm{N}$ fertilization management. Jiang, H. L. et al. (2015b) [21] showed that spectral indices can reduce or even eliminate the noise caused by environmental backgrounds such as soil and water bodies, which is higher than that of single band. The spectral index $\operatorname{mND}(780,712)$ proposed by J. Yang et al. (2009b) [22] introduces the reflectance of 445 $\mathrm{nm}$ band, thus reducing the effect of reflectance differences on the leaf surface and making the constructed model more application-friendly. Seventeen known spectral parameters were selected as the basis of this experiment, and by analyzing the quantitative relationships between these spectral parameters and the amount of chla, chlb and chla $+b$ containing chla $+b$, it was found that RVI $(729,755)$ and GREEN-NDVI were most closely related to the chla $+\mathrm{b}$ content. To identify the reliability of the model, the better chlorophyll content monitoring model was tested separately using hyperspectral data from the remaining sample sites at the same location, and the fitting accuracy (R2) was used as the test index, and a 1:1 relationship plot was used to describe the test effect of this inverse model more intuitively, and it was concluded that the wheat canopy chlorophyll content monitoring model with GREENNDVI as the variable was better and more reliable.

\subsubsection{Wheat leaf area index monitoring model}

LAI is an important indicator of wheat growth status and population structure, so conducting LAI monitoring is an important part of crop growth monitoring and yield assessment. There have been some studies on the construction of wheat canopy leaf area index monitoring models based on hyperspectral remote sensing, Liang Liang et al. (2011b) [23] and others compared and analyzed the differences of 18 hyperspectral indices on LAI response and found that although the spectral index NDVI 705 is commonly used for vegetation survey and monitoring, it is disturbed by atmospheric conditions, soil 
components, and the presence of dead material in the plant canopy itself ( Qietal. 1994), the spectral index OSAVI is relatively more sensitive to wheat LAI because it can overcome the influence of soil background and atmospheric conditions to a certain extent, and therefore can be used as the preferred index for wheat LAI inversion.

\subsubsection{Wheat seed yield prediction model}

Wheat is an important crop in China, and its yield is directly related to the food supply in China. The application of hyperspectral remote sensing technology in wheat yield prediction has received wide attention. Leaf area index (LAI) is one of the important indexes to characterize crop growth and development and predict yield, and a reasonable LAI is the basis for source reservoir coordination and balanced development of all parts of tissues and organs. Therefore, in this experiment, LAI was used as a linkage point to construct a prediction model based on canopy spectral data-LAI-seed yield of $\mathrm{Pu}$ Bing 151. Feng Wei (2008) [24] showed that the accumulation values of important spectral parameters in the pre-grazing period or characteristic spectral parameters from nodulation to maturity can be used to accurately and reliably predict the seed yield of different $\mathrm{N}$ applications and varieties in different years.

\section{References}

1. Zhao Chunjiang. Progress of agricultural remote sensing research and application[J]. Journal of Agricultural Machinery,2014,45(12):277-293.

2. Chen Zhongxin,Ren Jianqiang,Tang Huajun,et al. Progress and prospects of agricultural remote sensing research applications[J]. Journal of Remote Sensing,2016,20(5):748-767.

3. Shi Zhou,Liang Zongzheng,Yang Yuanyuan,et al. Current status and outlook of agricultural remote sensing research[J]. Journal of Agricultural Machinery,2015,46(2):247-260.

4. Xing Suli,Zhang Guanglu. Current situation and prospect of agricultural remote sensing application in China[J]. Journal of Agricultural Engineering,2003,19(6):174-178.

5. Wu BF, Zhang L, Li QZ, et al. Current status and prospects of agricultural and ecological remote sensing[J]. Journal of Remote Sensing,2009,13(s1):266-271.

6. Zhang Zengxiang, Wang Xiao, Wen Qingke, et al. Research progress of remote sensing application of land resources[J]. Journal of Remote Sensing,2016,20(5):1243-1258.

7. LIU Haiying,GAO Jixi,LI Zhenghai. Progress of remote sensing research on land cover and land use[J]. Remote Sensing of Land Resources, 2001(4):7-12.

8. Zhang Ruolin, Wan Li, Zhang Fawang, et al. Progress of land use remote sensing classification methods[J]. South-North Water Transfer and Water Conservancy Science and Technology,2006,4(2):39-42.
9. Tian M-L, Ban S-T, Chang Q-R, Ma W-J, Yin Z, Wang L. Inversion of chlorophyll content of cotton based on UAV imaging spectrometer data[J]. Journal of Agricultural Machinery,2016,47(11):285-293.

10. Yan Lin. Research on physiological and biochemical parameters of rice in Ningxia Yellow Irrigation Area based on hyperspectral remote sensing[D]. Northwest University of Agriculture and Forestry Science and Technology,2017.

11. Wang Ji-Hua,Huang Wen-Jiang,Laocai-Lian et al.2007.Inversion of nitrogen vertical distribution from wheat canopy reflectance spectra using PLS algorithm. Spectroscopy and Spectral Analysis,27(7):1319-1322.

12. Tong QX, Zhang B, Zhang LF. Frontier progress of hyperspectral remote sensing in China[J]. Journal of Remote Sensing,2016,20(05):689-707.

13. Fang Xiuqin,Zhang Wanchang.2003.A review of remote sensing quantitative methods for leaf area index (LAI). Remote Sensing of Land Resources, 15(3):58-62.

14. Hui Fengming, Tian Qingjiu, Jin Zhenyu, et al. 2003. Study on the relationship between vegetation index and leaf area index and quantitative analysis. Remote Sensing Information,(2):10-13.

15. Li Kaili, Jiang JJ, Mao RZ, et al. 2005. Remote sensing monitoring model of vegetation leaf area index. Journal of Ecology,25(6):1491-1496.

16. Xie Qiaoyun. Study on the generalizability of leaf area index inversion method [Master's thesis]. Anhui University,2014.

17. Wang Shuo. Research on the estimation of maize agronomic parameters in Guanzhong area based on hyperspectral remote sensing [D]. Northwest University of Agriculture and Forestry Science and Technology,2018.

18. Li D,He J,Liu Guishan et al.2014.Nondestructive detection of cucumber moisture based on hyperspectral imaging technology. Infrared and Laser Engineering,43(7):2393-2397.

19. Zhang Xiaoyan,Wang Lili,Liu Feng et al.2012. Research on monitoring model of peanut leaf nitrogen accumulation based on hyperspectral remote sensing Shandong Agricultural Science,44(3):712,16 .

20. Li Na. Construction of a model for monitoring agronomic parameters and seed protein content of wheat based on hyperspectral remote sensing[D]. Northwest University of Agriculture and Forestry Science and Technology,2017.

21. Jiang H L, Yang H,Chen S P,Wang Sh D,Li Xue Ke,Liu K,Cen Y. 2015.Research on the accuracy and stability of inversion of vegetation chlorophyll content using spectral indices. Spectroscopy and Spectral Analysis(04):975-981.

22. Yang J, Tian YC, Yao X, Cao Z, Zhang YS, Zhu Y. 2009a.Hyperspectral estimation model of chlorophyll 
content in upper leaves of rice. Journal of Ecology(12):6561-6571.

23. Liang L, Yang MH,Zhang LP,Lin $H$. 2011 b.Hyperspectral inversion of leaf area index of wheat. Spectroscopy and Spectral Analysis.(06):1658-1662.

24. Feng W,Zhu Y,Tian YC,Yao X,Guo TC,Cao Z.2007.Research on wheat seed yield prediction model based on hyperspectral remote sensing. Journal of Wheat Crops (06):1076-1084. 